\title{
A 3D Finite Radon Transform
}

\author{
Mustapha Boukour and Abderrahim El Omri \\ Faculté des Sciences et Techniques de Tanger, Laboratoire de Mécanique et Génie Civil, BP. 416 Tangier, Morocco
}

\begin{abstract}
The present work is devoted to the development of the extension of FRT (Finite Radon Transform) to the tridimensional case. One simple formulation is proposed and it is shown that it is the exact discretization of the continuous Discrete Radon Transform. More precisely this is the case when the sampling is given on a regular grid i.e. the continuous function is filtered by the mean of the box function. Relation of this transform with the Discrete Fourier one is given and is for some help in numerical implementation.
\end{abstract}

Key words: Radon transform, discrete radon transform, FRT, discrete Fourier, numerical computing.

\section{Introduction}

The Radon Transform proposed plays an important role in different domain of fundamental physics or image processing and image reconstructions [3, 4]. Different discretized formulations are proposed in Refs. $[5,8,9,10,12]$. The one of interest here is called Finite Radon Transform (FRT), e.g. [5]. Sometimes it is called Discrete Radon Transform [1, 2, 6, 7, 8]. It is well recognized $[10,12]$ that this discretized form is related in some way to the classical Radon Transform.

A continuous variant of the classical Radon Transform proposed in Ref. [11] is called a Discrete Radon Transform. It is also useful because it can be very used to solve some problem of divergence and curl free problems in physics and mechanics [13]. More precisely it has been shown that the problem of heterogeneous elasticity or conductivity can be dealt with this latter transform. To let this transform more effective, a discretization is needed to make the numerical implementation of this transform more effective. The FRT proposed in Ref. [5] appears to be an interesting and discrete alternative. However this latter is limited to the plane and its extension to the space $\mathbb{R}^{3}$ is required.

Corresponding author: Boukour Mustapha, PhD student, research fields: numerical computing, PDE solving, differential and integral geometry.
In Section 2 of this paper an FRT on $\mathbb{R}^{3}$ is proposed. As for the FRT in the two-dimensional case, we will be limited to tri dimensional $p \times p \times p$ matrix with $\mathrm{p}$ being a given prime number.

In Section 3, the obtained decomposition is related to the Discrete Fourier Transform. The obtained relations are to be useful for any numerical implementation. In the last section it is shown that the proposed FRT is the discretized form of the Discrete Radon Transform in $\mathbb{R}^{3}$.

\section{A Tridimensional FRT}

\subsection{Definition}

For every integer $m, n=0, p-1$, let us define by:

$$
\begin{gathered}
f_{0}=\frac{1}{p^{3}} \sum_{i, j, k=0}^{p-1} f(i, j, k) \\
\tilde{f}=f-f_{0} \\
f_{m, n}(x, y, z)=\frac{1}{p^{2}} \sum_{i, j=0}^{p-1} f(x+i, y+j, z+i m \\
+j n) \\
f_{p, n}(x, y, z)=\frac{1}{p^{2}} \sum_{i, j=0}^{p-1} f(x+i n, y+i, z+j) \\
f_{p+1}(x, y, z)=\frac{1}{p^{2}} \sum_{i, j=0}^{p-1} f(x+i, y, z+j)
\end{gathered}
$$




\subsection{Theorem}

Let $p$ be any prime number and let $f$ be a $p-$ periodic function defined on $\mathbb{R}^{3}$, i.e.:

$\forall(x, y, z) \in \mathbb{R}^{3}, \forall(m, n, r) \in \mathbb{Z}^{3}$

$$
f(x+m p, y+n p, z+r p)=f(x, y, z)
$$

The function $f$ can then be decomposed as:

$$
\begin{aligned}
f(x, y, z) & =\sum_{m, n=0}^{p-1} f_{m, n}(x, y, z)+\sum_{n=0}^{p-1} f_{p, n}(x, y, z) \\
& +f_{p+1}(x, y, z)-\left(p^{2}+p\right) f_{0}
\end{aligned}
$$

Proof: Let us first recall the FRT on the plane [5] for a $p$-periodic function:

$$
\begin{gathered}
g(x, y)=\frac{1}{p} \sum_{i, m=0}^{p-1} g(x+i, y+i m) \\
+\frac{1}{p} \sum_{i=0}^{p-1} g(x, i)-\frac{1}{p} \sum_{i, r=0}^{p-1} g(i, r)
\end{gathered}
$$

Using successively this relation relative to the couple $(x, y)$ or $\mathrm{e}(x, z)$ one can obtain easily:

$$
\begin{aligned}
f(x, y, z)=\frac{1}{p} \sum_{m, n=0}^{p-1} f_{m, n}(x, y, z) & \\
& +\frac{1}{p} \sum_{n=0}^{p-1} f_{p, n}(x, y, z)+f_{p+1}(x, y, z) \\
& -\left(p^{2}+p\right) f_{0}
\end{aligned}
$$

This completes the proof of the theorem. Moreover, it follows easily an important corollary more suitable for subsequent analysis.

\subsection{Corollary}

Let $p$ be any prime number and $f$ a $p$-periodic function defined on $\mathbb{R}^{3}$, i.e.:

$$
\begin{aligned}
& \forall(x, y, z) \in \mathbb{R}^{3} \text { and } \forall(m, n, r) \in \mathbb{Z}^{3} \\
& \quad f(x+m p, y+n p, z+r p)=f(x, y, z)
\end{aligned}
$$

By letting, $\tilde{f}=f-f_{0}$, one has:

$$
\begin{aligned}
f(x, y, z)=f_{0} & +\sum_{m, n=0}^{p-1} \tilde{f}_{m, n}(x, y, z)+ \\
& +\sum_{n=0}^{p-1} \tilde{f}_{p, n}(x, y, z)+\tilde{f}_{p+1}(x, y, z)
\end{aligned}
$$

\subsection{A Geometrical Interpretation}

As for the bi dimensional case we can note that each component of this transform of some real function is nothing else that it is mean in some specific plane.

Definition: Let us call a discrete plane passing by some point $\mathrm{A}$ and a vector $\vec{n} \in \mathbb{Z}^{3}$ it is discrete normal vector, the plane $\Pi(A, \vec{n})$ defined by:

$$
\Pi(A, \vec{n})=\{M:(x+i, y+j, z+k) / \overrightarrow{M A} \cdot \vec{n} \equiv 0[p]\}
$$

It can be noted that for every vector $\vec{n}$ element of

$$
[[0, p-1]]^{3}-\{(0,0,0)\} \text {, the plane } \Pi(A, \vec{n}) \text { is }
$$

finite and its cardinal is $p^{2}$. This allows defining $f_{\vec{n}}$ as a mean of $\tilde{f}$ on $\Pi(A, \vec{n})$. In other words:

$$
f_{\vec{n}}(x, y, z)=\frac{1}{p^{2}} \sum_{M \in \Pi(A, \vec{n})} \tilde{f}(M)
$$

On another hand, by letting:

$$
\begin{gathered}
\mathcal{N}=\{(m, n, 1) / m, n=0 . . p-1\} \cup \\
\{(m, 1, n) / m=0 . . p-1\} \cup\{(1,0,0)\}
\end{gathered}
$$

it comes that to every vector $\vec{n}$ such that:

$$
\vec{n} \in[[0, p-1]]^{3}-\{(0,0,0)\},
$$

corresponds one and only one vector $\overrightarrow{n^{\prime}} \in \mathcal{N}$ such that $\Pi(A, \vec{n})=\Pi\left(A, \overrightarrow{n^{\prime}}\right)$.

In other words, it can be said that the map:

$$
\left\{\begin{array}{c}
{[[0, p-1]]^{3}-\{(0,0,0)\} \rightarrow \mathcal{N}} \\
\vec{n} \rightarrow \overrightarrow{n^{\prime}} / \Pi(A, \vec{n})=\Pi\left(A, \overrightarrow{n^{\prime}}\right)
\end{array}\right.
$$

is surjective and every element of $\mathcal{N}$ is paired with $p-1$ elements.

We can now present the 3D FRT in a more suitable form:

$$
f(x, y, z)=f_{0}+\sum_{\vec{n} \in \mathcal{N}} f_{\vec{n}}(x, y, z)
$$

\section{From the Tridimensional Discrete Radon Transform to the FRT}

\subsection{The 3D Discrete Radon Transform}

An extension of the Discrete Radon Transform [11, 
13] of a integrable function $f$ defined in $[0,1]^{3}$, reads:

$$
f(x)=f_{0}+\sum_{\vec{n} \in \mathbb{A}} f^{\vec{n}}(\vec{x} \cdot \vec{n})
$$

with

$$
\begin{aligned}
& f_{0}=\int_{\mathbb{R}^{3}} f(\vec{x}) d \vec{x} \tilde{f}=f-f_{0} \\
& f^{\vec{n}}(\vec{x} \cdot \vec{n})=T^{\vec{n}} \sum_{r \in \mathbb{Z}} \mathcal{R}^{\vec{n}} f\left(\rho+r T^{\vec{n}}\right) \\
& \mathcal{R}^{\vec{n}} f(\vec{x} \cdot \vec{n})=\iiint_{\mathbb{R}^{3}} f\left(\overrightarrow{x^{\prime}} \cdot \vec{n}\right) \delta\left(\overrightarrow{x^{\prime}} \cdot \vec{n}-\rho\right) d \overrightarrow{x^{\prime}} \\
& \mathbb{A}=\left\{\vec{n}=\frac{\left(n_{1}, n_{2}, n_{3}\right)}{\sqrt{n_{1}^{2}+n_{2}^{2}+n_{3}^{2}}},\right. \\
& \left(n_{1}, n_{2}, n_{3}\right) \in \mathbb{Z}^{3}-\{(0,0,0)\} /\left(\left(n_{1}>0\right)\right. \\
& \text { or } \left.\left.\left(n_{1}=0 \text { and } n_{2}>0\right)\right) \text { and }\left(n_{1} \wedge n_{2} \wedge n_{3}=1\right)\right\} \\
& \cup\{(0,0,1)\}
\end{aligned}
$$

\subsection{A Sampling of the $3 D D R T$}

The previous transform will now be related to the 3D FRT proposed in this work (9).

More precisely if a function is sampled in on a regular grid, the 3D FRT is a discretized form of the 3D DRT.

Let $f$ be a real and integrable function defined on $[0,1]^{3}$ and let $I I I_{p}$ be the tridimensional Shah function:

$$
\begin{gathered}
I I I_{p}(x, y, z)=\frac{1}{p^{3}} \sum_{i, j, k \in \mathbb{Z}}\left(\delta\left(x-\frac{i}{p}\right)\right. \\
\left.\delta\left(y-\frac{j}{p}\right) \delta\left(z-\frac{k}{p}\right)\right)
\end{gathered}
$$

A regular and discrete sampling $f_{d}$ of the function $f$ is hence obtained by:

$$
\begin{gathered}
f_{d}=f I I_{p} \\
f_{d}(x, y, z)= \\
=\frac{1}{p^{3}} \sum_{i, j, k \in \mathbb{Z}} f\left(\frac{i}{p}, \frac{j}{p}, \frac{k}{p}\right) \delta\left(x-\frac{i}{p}\right) \delta\left(y-\frac{j}{p}\right) \delta\left(z-\frac{k}{p}\right)
\end{gathered}
$$

Using a filter function $\phi$ the filtered function $f_{\phi}$ of $f$ is obtained classically using the convolution product:

$$
f_{\phi}=f_{d} * \phi
$$

$$
\begin{array}{r}
f_{\phi}(x, y, z)=\frac{1}{p^{3}} \sum_{i, j, k \in \mathbb{Z}}\left(f\left(\frac{i}{p}, \frac{j}{p}, \frac{k}{p}\right)\right. \\
\left.\phi\left(x-\frac{i}{p}\right) \phi\left(y-\frac{j}{p}\right) \phi\left(z-\frac{k}{p}\right)\right)(22)
\end{array}
$$

The Discrete Radon Transform (15) of $f_{\phi}$ in the direction $\vec{n} \in \mathbb{A}$ is given by:

$$
f_{\phi}^{\vec{n}}(\rho)=T^{\vec{n}} \sum_{r \in \mathbb{Z}} \mathcal{R}^{\vec{n}} \tilde{f}_{\phi}\left(\rho+r T^{\vec{n}}\right)
$$

with

$$
\begin{gathered}
\mathcal{R}^{\vec{n}} \tilde{f}_{\phi}(\rho)=\frac{1}{p^{3}} \sum_{i, j, k \in \mathbb{Z}}^{+\infty}\left(\tilde{f}\left(\frac{i}{p}, \frac{j}{p}, \frac{k}{p}\right)\right. \\
\iiint_{\mathbb{R}^{3}} \phi\left(x^{\prime}-\frac{i}{p^{\prime}}, y^{\prime}-\frac{j}{p}, z^{\prime}-\frac{k}{p}\right) \delta\left(n_{1} x^{\prime}+n_{2} y^{\prime}\right. \\
\left.\left.+n_{3} z^{\prime}\right) d x^{\prime} d y^{\prime} d z^{\prime}\right)
\end{gathered}
$$

Let us now take as a filter function $\phi$ the box function is defined by :

$$
\phi(x, y, z)=\Delta(x) \Delta(y) \Delta(z)=\left\{\begin{array}{l}
1 \text { if }|x|<0 \\
0 \text { elsewhere }
\end{array}\right.
$$

and let us denote by:

$$
\begin{gathered}
\vec{n}=\frac{(m, n, 1)}{\sqrt{m^{2}+n^{2}+1}} \text { and } A=\left(\frac{i_{0}}{p}, \frac{j_{0}}{p}, \frac{k_{0}}{p}\right) \\
\rho=\overrightarrow{O A} \cdot \vec{n}=\frac{m i_{0}+m j_{0}+k_{0}}{p \sqrt{m^{2}+n^{2}+1}}
\end{gathered}
$$

Eq. (23) can hence be written as:

$$
\begin{array}{r}
f_{\phi}^{\vec{n}}(\rho)=\frac{1}{p^{2}} \sum_{i, j, k, r}^{+\infty} \tilde{f} \tilde{f}\left(\frac{i}{p}, \frac{j}{p}, \frac{k}{p}\right) \Delta\left(m\left(i-i_{0}\right)\right. \\
\left.+n\left(j-j_{0}\right)+\left(k-k_{0}\right)-p r\right)
\end{array}
$$

which is nothing else than:

$$
f_{\phi}^{\vec{n}}(\rho)=\frac{1}{p^{2}} \sum_{M \in \Pi(A, \vec{n})} \tilde{f}(M)
$$

So the sampled and filtered form $f_{\phi}^{\vec{n}}$ is an FRT component in one direction: 


$$
f_{\phi}^{\vec{n}}(\rho)=f_{\vec{n}}\left(\frac{i_{0}}{p}, \frac{j_{0}}{p}, \frac{k_{0}}{p}\right)
$$

\section{FRT and Discrete Fourier Transform}

To rely the FRT to the Discrete Fourier Transform DFT, three different cases are to be considered. Let us recall that the FRT of a $p^{3}$-matrix with $p$ any given prime number:

$$
A=A_{0}+\sum_{\vec{n} \in \mathcal{N}} \bar{A}^{\vec{n}}
$$

With

$$
\begin{gathered}
A_{0}=\frac{1}{p^{3}} \sum_{i, j, k=0}^{p} A(i, j, k) \\
\tilde{A}=A-A_{0} \\
\bar{A}^{\vec{n}}(i, j, k)=\frac{1}{p^{2}} \sum_{(q, r, s) \in \Pi(i, j, k, \vec{n})} \tilde{A}(q, r, s)
\end{gathered}
$$

4.1 A Discrete Plane Based Decomposition of a $p^{3}$ Matrix

Before evaluating the DFT of the different components of the FRT a decomposition based on the discrete planes defined previously (25) is developed. Let us recall the classical Hadamard product of two matrices $A$ and $B$ :

$$
(A \underline{\times} B)(i, j, k)=A(i, j, k) B(i, j, k)
$$

For each direction $\vec{n}=(i, j, k)$ corresponds a matrix:

$$
O^{\vec{n}}(q, r, s)=\left\{\begin{array}{c}
0 \text { if } \vec{n} \times(q, r, s) \neq(0,0,0)[p] \\
1 \text { if not }
\end{array}\right.
$$

In particular:

$$
\text { If } \begin{aligned}
\vec{n}=(i, j, 1), \\
\quad \begin{aligned}
O^{\vec{n}}(q, r, s) \\
=
\end{aligned} \\
\begin{array}{r}
\text { if }(j s-q \neq 0[p]) \operatorname{or}(i s-r \neq 0[p]) \\
\text { if not }
\end{array}
\end{aligned}
$$

If $\vec{n}=(i, j, 0)$,

$O^{\vec{n}}(q, r, s)=\left\{\begin{array}{c}0 \text { if }(i s \neq 0[p]) \operatorname{or}(j s \neq 0[p]) \\ 1 \text { if not }\end{array}\right.$

If $\vec{n}=(i, 0,0)$,

$$
O^{\vec{n}}(q, r, s)=\left\{\begin{array}{c}
0 \text { if }(s \neq 0[p]) \text { or }(r \neq 0) \\
1 \text { if not }
\end{array}\right.
$$

Hence, every $p^{3}$ matrix verifying $(A(0,0,0)=0)$ can be decomposed into the following form:

$$
A=\sum_{\vec{n} \in \mathcal{N}} O^{\vec{n}} \times A
$$

verifying the orthogonal property:

$$
\begin{gathered}
\forall(\vec{n}, \vec{m}) \in \mathcal{N}^{2}, \forall(q, r, s) \neq(0,0,0) \\
O^{\vec{n}} \underline{x} O^{\vec{m}}=\delta_{\vec{n}, \vec{m}} O^{\vec{n}}(q, r, s)
\end{gathered}
$$

Also if $A(0,0,0)=0$ one has

$$
\forall(\vec{n}, \vec{m}) \in \mathcal{N}^{2}, O^{\vec{n}} \times O^{\vec{m}} \times A=\delta_{\vec{n}, \vec{m}} O^{\vec{n}} \times A
$$

\subsection{DFT of the FRT Components}

Let us define by $\widehat{A}$ as a DFT of a $p^{3}$ matrix $A$ with vanishing mean:

$$
\widehat{A}(i, j k)=\sum_{q, r, s=0}^{p-1} A(q, r, s) w^{-(i q+j r+k s)}
$$

with $w=e^{\frac{2 I \pi}{p}}$ and $I$ the complex number $\left(I^{2}=-1\right)$.

The inverse DFT of $A$ is denoted by $F T^{-1} A$ :

$$
\begin{gathered}
\left(D F T^{-1} A\right)(i, j, k)= \\
\frac{1}{p^{2}} \sum_{q, r, s=0}^{p-1} A(q, r, s) w^{(i q+j r+k s)}
\end{gathered}
$$

To evaluate the DFT DFT $\widehat{\bar{A}}$ of each FRT component $\bar{A}^{\vec{n}}$ of $A(28,37)$, three cases will be considered:

Case 1: $\vec{n}=(m, n, 1)$

In this case the DFT is given by:

$$
\widehat{\bar{A}^{n}}=\left\{\begin{array}{c}
i f(i-k m \neq 0[p]) \operatorname{or}(j-k n \neq 0[p]) \\
p^{2} \sum_{s=0}^{p-1} A(0,0, s) w^{-k s}
\end{array}\right.
$$

Proof: Let $A=A(i, j, k), \quad(i, j, k=0 . . p-1)$ constant in each discrete $\Pi^{\vec{n}}(M)$ with $\vec{n}=(m, n, 1)$ and $M=(0,0, s)$ i.e.:

$$
A(0,0, s)=A\left(q, r,[s-q m-r n]_{p}\right.
$$


Because the following map

$$
\left\{\begin{array}{c}
{[[0, p-1]]^{3} \rightarrow[[0, p-1]]^{3}} \\
(q, r, s) \rightarrow\left(\mathrm{q}, \mathrm{r},[\mathrm{s}-\mathrm{qm}-\mathrm{rn}]_{\mathrm{p}}\right)
\end{array}\right.
$$

is bijective, so:

$$
\begin{aligned}
& B(i, j, k)=\sum_{q, r, s=0}^{p-1} A(q, r, s) w^{-(i q+j r+k s)} \\
& B(i, j, k)=\sum_{q, r, s=0}^{p-1}\left(A\left(q, r,[s-q m-r n]_{p}\right)\right. \\
&\left.w^{-\left(i q+j r+k[s-q m-r n]_{p}\right)}\right)
\end{aligned}
$$

Because $w^{p}=1$ and by a change of indices one has:

$$
\begin{gathered}
B(i, j, k)= \\
\sum_{s=0}^{p-1} A(0,0, s) w^{-k s} \sum_{q=0}^{p-1}\left(w^{-(i-k m)}\right)^{q} \sum_{q=0}^{p-1}\left(w^{-(j-k n)}\right)^{r}
\end{gathered}
$$

which leads finally to Eq. (40).

Case 2: $\vec{n}=(m, 1,0)$

This case corresponds to the FRT in the two dimensional case. One can obtain similarly as before:

$$
\widehat{\vec{A}}=\left\{\begin{array}{c}
0 \text { if }(i-j m \neq 0[p]) \text { or }(k \neq 0[p]) \\
p^{2} \sum_{r=0}^{p-1} A(0, r, 0) w^{-k s} \text { if not }
\end{array}\right.
$$

Case 3: $\vec{n}=(1,0,0)$

The corresponding DFT to the matrix A is also given by:

$$
\widehat{\bar{A}}=\left\{\begin{array}{c}
0 \text { if }(j \neq 0[p]) \text { or }(k \neq 0[p]) \\
p^{2} \sum_{r=0}^{p-1} A(q, 0,0) w^{-k s} \text { if not }
\end{array}\right.
$$

It follows that the DFT of every component of the FRT of a matrix A can be deduced from the DFT of the matrix $A$ using the projectors defined in Eq. (30) via the following result.

\section{Lemma 1:}

Let the $p^{3}$-matrix $\mathrm{A}$ with $p$ any given prime number. The DFT of $\bar{A}^{\vec{n}}$ for every $\vec{n} \in \mathcal{N}$, writes:

$$
\widehat{\vec{A}}=O^{\vec{n}} \times \widehat{A}
$$

Proof: By noting from Eqs. (31), (39)-(41) that

$$
\overrightarrow{\bar{A}}=O^{\vec{n}} \times \overrightarrow{\bar{A}}
$$

using the linearity of the DFT and Eq. (36) one has easily:

$$
\begin{gathered}
O^{\vec{m}} \times \hat{A}=\sum_{\vec{n} \in \mathcal{N}} O^{\vec{m}} \times O^{\vec{n}} \underline{\widehat{A}} \widehat{\vec{n}} \\
=O^{\vec{m}} \times \widehat{\vec{A}}=\widehat{\vec{A}}
\end{gathered}
$$

It follows directly from this result, the relation between the DFT and FRT.

Lemma 2:

Let $A$ be a $p^{3}$-matrix with $p$ a prime number and let $\hat{A}$ be the DFT of $A$. The components of the FRT of $A$ are given by:

$$
\forall \vec{n} \in \mathcal{N}, \vec{A}^{\vec{n}}=T F D^{-1}\left(O^{\vec{n}} \underline{\hat{A}}\right)
$$

\section{Conclusion}

An extension of the classical FRT to the tri dimensional case is presented. Hence a $p^{3}$ matrix with $p$ a prime number can be decomposed as simply as in the two dimensional case.

It has also been shown by using the box function as a filter that this transform is the discretization of the tri dimensional Discrete Radon Transform. The analysis of the Discrete Fourier Transform of these projections is laid down and the obtained results presented here are nothing else than the expression of the classical slice theorem used for the classical Radon Transform. This allows the complexity of the algorithm required to evaluate each projection to be the same as for the Discrete Fourier Transform. The properties presented in the last section are more useful essentially for the case where a linear combination of FRT is needed to be implemented.

\section{References}

[1] Beylkin, G. 1984. "The Inversion Problem and Applications of the Generalized Radon Transform." 
Commun. Pure Appl. Math. 37: 579-99.

[2] Beylkin, G. 1987. "Discrete Radon Transform." IEEE Trans. Acoustics, Speech, Signal Process 35: 162-72.

[3] Natterer, F. 1989. The Mathematics of Computerized Tomography. Wiley.

[4] Deans, S. R. 1993. The Radon Transform and Some of Its Applications, revised ed. Melbourne, FL: Krieger Publishing Company.

[5] Matus, F., and Flusser, J. 1993. "Image Representation via a Finite Radon Transform.” IEEE Trans. Pattern Anal. Machine Intell. 15: 996-1006.

[6] Kelley, B. T., and Madisetti, V. K. 1993. "The Fast Discrete Radon Transform. I. Theory." IEEE Trans. Image Process 2: 382-400.

[7] Gotz, W. A., and Druckmuller, H. J. 1995. "A Fast Digital Radon Transform: An Efficient Means for Evaluating the Hough Transform." Pattern Recognition 28: 1985-92.

[8] Hsung, T. C., Lun, D. P. K., and Siu, W. C. 1996. "The Discrete Periodic Radon Transform.” IEEE Trans. Signal
Process 44: 2651-7.

[9] Brady, M. L. 1998. "A Fast Discrete Approximation Algorithm for the Radon Transform." SIAM J. Comput. 27: 107-19.

[10] Averbuch, A., and Shkolnisky, Y. 2003. "3D Fourier Based Discrete Radon Transform." Appl. Comput. Harmon. Anal. 15: 33-69.

[11] Gelfand, I. M., Gindikin, S. G., and Graev, M. I. 2003. Selected Topics in Integral Geometry. AMS Providence, Rhode Island.

[12] Averbuch, A., Coifman, R. R., Donohos, D. L., Israeli, M., Shkolnisky, Y., and Sedelnikov, I. 2008. "A Framework for Discrete Integral Transformations II, The 2D Discrete Radon Transform.” SIAM J. Sci. Comput. 30 (2): 785-803.

[13] Boukour, M., and Omri, A. "Solving the Problems of the Conductivity of Heterogeneous Media by the Method of the Discrete Radon Transform." Presented at Communication in 3rd Spring School on Numerical Methods for Partial Differential Equations, 17-21 April 2018, Morocco. 